\title{
Protein-driven lipid domain nucleation in biological membranes
}

\author{
Moritz Hoferer, ${ }^{1}$ Silvia Bonfanti, ${ }^{2}$ Alessandro Taloni, ${ }^{3}$ Caterina A. M. La Porta, ${ }^{4,5}$ and Stefano Zapperi ${ }^{2,6, *}$ \\ ${ }^{1}$ ETH Zurich, Zürichbergstrasse 18, 8092 Zurich, Switzerland \\ ${ }^{2}$ Center for Complexity and Biosystems, Department of Physics, University of Milan, Via Celoria 16, 20133 Milano, Italy \\ ${ }^{3}$ CNR - Consiglio Nazionale delle Ricerche, Istituto dei Sistemi Complessi, via dei Taurini 19, 00185 Roma, Italy \\ ${ }^{4}$ Center for Complexity and Biosystems, Department of Environmental Science and Policy, University of Milan, via Celoria 26, \\ 20133 Milano, Italy \\ ${ }^{5}$ CNR - Consiglio Nazionale delle Ricerche, Istituto di Biofisica, Via Celoria 26, 20133 Milano, Italy \\ ${ }^{6}$ CNR - Consiglio Nazionale delle Ricerche, Istituto di Chimica della Materia Condensata e di Tecnologie per l'Energia, Via R. Cozzi 53, \\ 20125 Milano, Italy
}

(Received 8 May 2019; revised manuscript received 10 September 2019; published 18 October 2019)

\begin{abstract}
Lipid rafts are heterogeneous dynamic lipid domains of the cell membranes that are involved in several biological processes, such as protein and lipid specific transport and signaling. Our understanding of lipid raft formation is still limited due to the transient and elusive nature of these domains in vivo, in contrast with the stable phase-separated domains observed in artificial membranes. Inspired by experimental findings highlighting the relevance of transmembrane proteins for lipid rafts, we investigate lipid domain nucleation by coarse-grained molecular dynamics and Ising-model simulations. We find that the presence of a transmembrane protein can trigger lipid domain nucleation in a flat membrane from an otherwise mixed lipid phase. Furthermore, we study the role of the lipid domain in the diffusion of the protein showing that its mobility is hindered by the presence of the raft. The results of our coarse-grained molecular-dynamics and Ising-model simulations thus coherently support the important role played by transmembrane proteins in lipid domain formation and stability.
\end{abstract}

DOI: 10.1103/PhysRevE.100.042410

\section{INTRODUCTION}

Biological membranes are ubiquitous cell structures that play crucial roles for the regulation of many vital processes, such as selective permeability and homeostasis maintenance, signaling and ion conductivity [1,2]. Membranes display a huge complexity in their microscopic structure, being composed of more than one thousand different lipids, cholesterol, several types of proteins, and a small number of carbohydrates. In an aqueous solvent, lipids spontaneously arrange in a double layer (with hydrophilic heads pointing outside the sheet and hydrophobic tails pointing inside the sheet) that can forge different structures depending on the environment, such as flat membranes or spherical and cylindrical micelles.

An intriguing feature of membranes is the emergence of lipid rafts, highly dynamic lateral heterogeneous domains (in mobility as well as formation and disruption) [3] at different length scales $(\sim \mathrm{nm})$ and timescales $(\sim \mathrm{ms})[4,5]$. Rafts consist in aggregates of saturated lipids and proteins such as glycosylphosphatidylinositol-anchored (GPI-anchored) or transmembrane proteins (see Fig. 1), induced by proteinprotein and protein-lipid interactions and are thought to be responsible for many membrane-associated functions [6-17]. Recent advances in experimental detection techniques [18], such as fluorescence microscopy [19], super-resolution optical microscopy [20], interferometric scattering microscopy [21],

\footnotetext{
*stefano.zapperi@unimi.it
}

single-particle tracking [22-24], and Forster resonance energy transfer (FRET) [25] contribute to a deeper understanding of membrane lateral heterogeneity and functions. However, the nature of lipid rafts and the mechanisms at the origin of their formation are still debated [3]. The major experimental challenge remains their direct unambiguous microscopic detection in vivo, which involves highly complex short-lifetime nanoscale processes.

It has been often speculated that lateral heterogeneities in membranes are caused by the separation of lipids in two coexisting domains with different degrees of order (liquidordered, with high packing, and liquid disordered), lateral diffusivity, and composition $[3,27,28]$. This behavior has been observed in artificial membrane model systems, from simple bilayers [29] with few proteins to vesicles [30,31] up to giant protein-rich membranes [32]. Phase-separated domains are, however, not representative of rafts in vivo, which occur in a mixed phase, so that a direct translation of the results obtained in artificial membranes to living cell membranes remains a major challenge. Model membranes dramatically differs from living membranes, mainly in composition and environmental conditions: Model membranes are at equilibrium and the phase domain partitions strongly depend on the preparation protocol $[3,25,33,34]$.

Note that, in the present work, only flat lipid membranes are discussed and studied. Biological membranes, however, are often curved on different length scales [35] and it has been widely shown-experimentally on artificial [36] and supported membranes [37] and theoretically via numerical modeling [38,39] — that membrane curvature can control the 


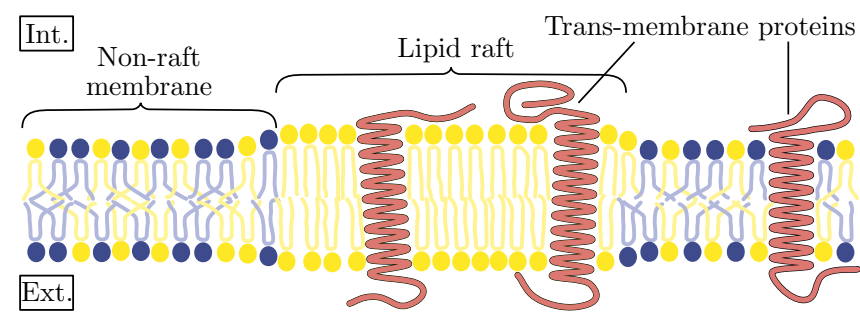

FIG. 1. Sketch of lipid raft domain in a cell membrane. Raft regions are composed of raft lipids (yellow) and transmembrane proteins (red). Non-raft regions of the membrane are characterized by non-raft-lipids (blue), raft lipids, and proteins in a mixed phase. "Int." ("Ext.") indicates the intracellular (extracellular) space. The figure is adapted from Ref. [26]; CC BY-SA 2.5 license.

geometry, size, and spatial organization of lipid rafts or microdomains, as discussed, e.g., in Ref. [40].

Several theoretical models have been proposed to explain the nanoscale lateral inhomogeneities in flat membranes. A well-known theoretical scenario [41-47] describes the phase transition from liquid order to liquid disorder depending on temperature in biomimetic membranes, stating that the cell membrane composition is tuned in the proximity of a critical point, where fluctuations typical of two-dimensional (2D) Ising-like phenomena are observed. Other proposed models consider the constitution of micro-emulsions [48], or the presence of nano- to micro-sized domains in the membrane [49-51], or three-component fluid mixtures that contain an active agent [33]. Further approaches examine the role of the underlying cytocortex and the actin cytoskeleton [52-55] in the raft organization.

To shed light on the mechanism leading to the raft formation in vivo, we perform extensive computer simulations using a molecular-dynamics (MD) coarse-grained model proposed by Deserno et al. [56] and an Ising-based model [44,46,47]. The advantages of this approach are the direct investigation of raft dynamics, in terms of stability and mobility, and the possibility to explore a wide range of timescales and length scales comparing two different methods.

To reproduce living membrane conditions, we set both models in a mixed phase, in the proximity of the transition point, carefully checking for the absence of alreadyphase-separated domains. We find that the introduction of a transmembrane raft protein, interacting with membrane lipids, triggers the lipids nucleating a local phase separation, in agreement with recent experimental results in living systems $[57,58]$. We study the evolution of the raft local domain in terms of average cluster size as a function the variables of the models, like temperature and interaction strength among proteins and lipids. The stability of the average cluster size together with the diffusive motion of the protein observed through the analysis of the mean squared displacement (MSD) indicates that the formation of the raft occurs in the liquid phase.

From real-space distribution calculations, we observe that the additional protein drives the aggregation behavior of the raft. Our analysis, combining different timescales and length scales - coarse-grained simulations are limited compared with an Ising-like approach—suggests the fundamental role of transmembrane proteins in the raft formation near the transition point. In analogy with the Ising model, far from the transition line, when the degree of the disorder is too strong, the correlation length is small and therefore the protein is not able induce raft formation. Close to the critical point, however, small perturbations such as those introduced by the raft protein can lead to large changes and trigger the formation of a lipid raft.

The paper is organized as follows: In Sec. II, we describe our simulation models and their computational setting. In Sec. III, we show our numerical results. In Sec. IV, we conclude.

\section{COMPUTATIONAL METHODS}

To describe the membrane dynamics in a general way, we distinguish between "raft lipids" (R), which are those that have the tendency to aggregate in raft and "nonraft lipids" (N) that do not assemble in aggregates. The addition of a transmembrane raft protein $(\mathrm{P})$, interacting with both types of membrane lipids, i.e., $\mathrm{R}$ and $\mathrm{N}$, triggers a local phase separation in the form of lipid clusterization. Both systems are binary mixtures of lipids, $\mathrm{R}$ and $\mathrm{N}$, in a $1: 7$ concentration ratio. Note that the protein is attractive to the minority phase (e.g., the raft lipids). The concentration ratio used here is similar to those used in experimental studies on domain emergence in model membranes. If we wish to compare our models with cellular membranes considering cholesterol as the main actor in lipid raft formation, the ratio $1: 7$ is too small to describe the real situation in plasma membranes of mammalian cells, but might be more relevant for the endoplasmic reticulum or the Golgi apparatus.

\section{A. Molecular-dynamics simulations}

For the molecular-dynamics model, we simulate a twocomponent bilayer membrane using the solvent-free model introduced by Deserno et al. [56], already employed to reproduce self-assembly, raft formation, melting, and fusion of single- and multicomponent bilayer membranes [56,59]. In this model, a lipid is composed of three beads, one representing the hydrophilic head and two representing the hydrophobic tail. We model the interactions in reduced Lennard-Jones units, where $\sigma$ is the unit of length, $\epsilon$ is the unit of energy, $m$ is the mass, and time is in units $\tau=\sqrt{\frac{\epsilon}{m \sigma^{2}}}$ [60]. The simulations are performed with the LAMMPS MD simulator [61] and the relevant scripts are available on GitHub [62]. Following previous numerical work [59], one can map the units of the model into physical units, taking into account the characteristic scales of lipid bilayers: Considering a membrane thickness of about $5 \mathrm{~nm}$, one has $\sigma \sim 0.5 \mathrm{~nm}$; the diffusion coefficient in the fluid phase is $\sim 1 \mu \mathrm{m}^{2} \mathrm{~s}^{-1}$, then $\tau \sim 10 \mathrm{~ns}[56,63]$. Considering the transition between ordered and disordered fluid states around $315 \mathrm{~K}$, one can set $\epsilon \sim 4.6 \mathrm{~kJ} \mathrm{~mol}^{-1}[56,59,64]$.

The total interaction potential is given by the sum of four terms: (i) A potential acting between head-head, head-tail, and tail-tail given by $V(r, b)=4 \epsilon\left[(b / r)^{12}-(b / r)^{6}+1 / 4\right]$ with the following set of parameters: $b_{\text {tail,tail }}=\sigma, b_{\text {head,head }}=$ $b_{\text {head,tail }}=0.95 \sigma$. These potentials are truncated at a distance $r_{\mathrm{c}}=2^{1 / 6} b$. (ii) A finite extensible nonlinear elastic (FENE) 
potential, keeping together the three beads: $V_{\text {bond }}(r)=$ $-1 / 2 k_{\text {bond }} r_{\infty}^{2} \ln \left[1-\left(r / r_{\infty}\right)^{2}\right]$, where $k_{\text {bond }}=30 \epsilon / \sigma^{2}$ is the stiffness and the divergence length $r_{\infty}=1.5 \sigma$. (iii) A harmonic spring potential with rest length $4 \sigma$ between the head and the lower tail bead: $V_{\text {bend }}(r)=1 / 2 k_{\text {bend }}(r-4 \sigma)^{2}$. (iv) An attractive potential that only affects the tail-tail interaction, given by

$$
V_{\mathrm{attr}}(r)= \begin{cases}-\epsilon & \left(r<r_{\mathrm{c}}\right) \\ -\epsilon \cos ^{2}\left(\frac{\pi\left(r-r_{\mathrm{c}}\right)}{2 w_{\mathrm{c}}}\right) & \left(r_{\mathrm{c}} \leqslant r \leqslant r_{\mathrm{c}}+w_{\mathrm{c}}\right) \\ 0 & \left(r>r_{\mathrm{c}}+w_{\mathrm{c}}\right),\end{cases}
$$

where $w_{\mathrm{c}}$ is the potential width, the key tunable parameter, together with the temperature, determining the phase state of the lipid species in the membrane [56]. The tail-tail potential has an attractive regime depending on the potential width $w_{\mathrm{c}}$.

We perform equilibration runs and prepare the system with the two types of lipids in a mixed fluid phase by tuning the potential widths $w_{\mathrm{c}}^{\mathrm{RR}}=w_{\mathrm{c}}^{\mathrm{NN}}=1.8 \sigma$ and $w_{\mathrm{c}}^{\mathrm{RN}}=1.75 \sigma$. Note that increasing the difference between the potential widths $w_{\mathrm{c}}^{\mathrm{RR}, \mathrm{NN}}$ and $w_{\mathrm{c}}^{\mathrm{RN}}$ leads to phase separation of the two types of lipids; not suitable to represent physiological membrane conditions. We simulate a double layer with 4100 lipids in a box size with periodic boundary conditions along $x$ and $y$ in an NpT ensemble, controlled by a Nosé-Hoover thermostat. The target pressure is zero. We investigate four different temperatures in the liquid phase, from 1.10 to 1.25 (reduced units). Note that the range of temperature is limited to preserve the stability of the membrane and the mixed-phase condition.

The lateral view of the bilayer structure, reported in Fig. 2(a), shows raft lipids with yellow beads and non-raft lipids with blue beads. The heads (marked with dark yellow and dark blue) point outside the leaflet while the tails face toward the center. The top view of the membrane in the mixed phase at $T=1.10$ is presented in Fig. 2(b).

After equilibration runs, we introduce an additional transmembrane protein that is attractive to raft lipids and lead to their local clusterization. The protein interacts with both types of lipids with a simple Lennard-Jones potential, as done elsewhere [65]: $V_{\mathrm{LJ}}(r)=4 \bar{\epsilon}\left[\left(\frac{1.2 \sigma}{r}\right)^{12}-\left(\frac{1.2 \sigma}{r}\right)^{6}\right]$ for $r \leqslant$ $r_{\mathrm{c}}$. The depth of the potential well is $\bar{\epsilon}=2$ and the cutoff distance $r_{\mathrm{c}}=6.0 \sigma$ for the interaction with lipids of type $\mathrm{R}$, while $\bar{\epsilon}=0.05$ and $r_{\mathrm{c}}=2.0 \sigma$ for the interaction with lipids of type $\mathrm{N}$. We model the protein with 78 beads arranged in a face-centered cubic structure with a hourglass shape, so that the two extremes have a radius of $\sim 2.5 \sigma$ that linearly reduces to $\sim 1.0 \sigma$ at the center. The lateral extension of the protein $(\sim 7 \sigma)$ is slightly bigger than the thickness of the membrane $(\sim 6 \sigma)$, in agreement with previous work [65]. The lateral and top views of the protein embedded in the membrane, triggering a raft domain, are reported in Figs. 2(c) and 2(d) for the system at $T=1.10$. The beads constituting the protein are represented in red and are treated as a single rigid body, i.e., they maintain their relative position throughout the MD dynamics. Therefore, the protein egg timer shape is always conserved. Simulations are performed for a membrane composed of 40000 lipids. The evolution dynamics of the protein-driven lipid raft domain at $T=1.10$ is shown in the Supplemental Movie S1 [66].

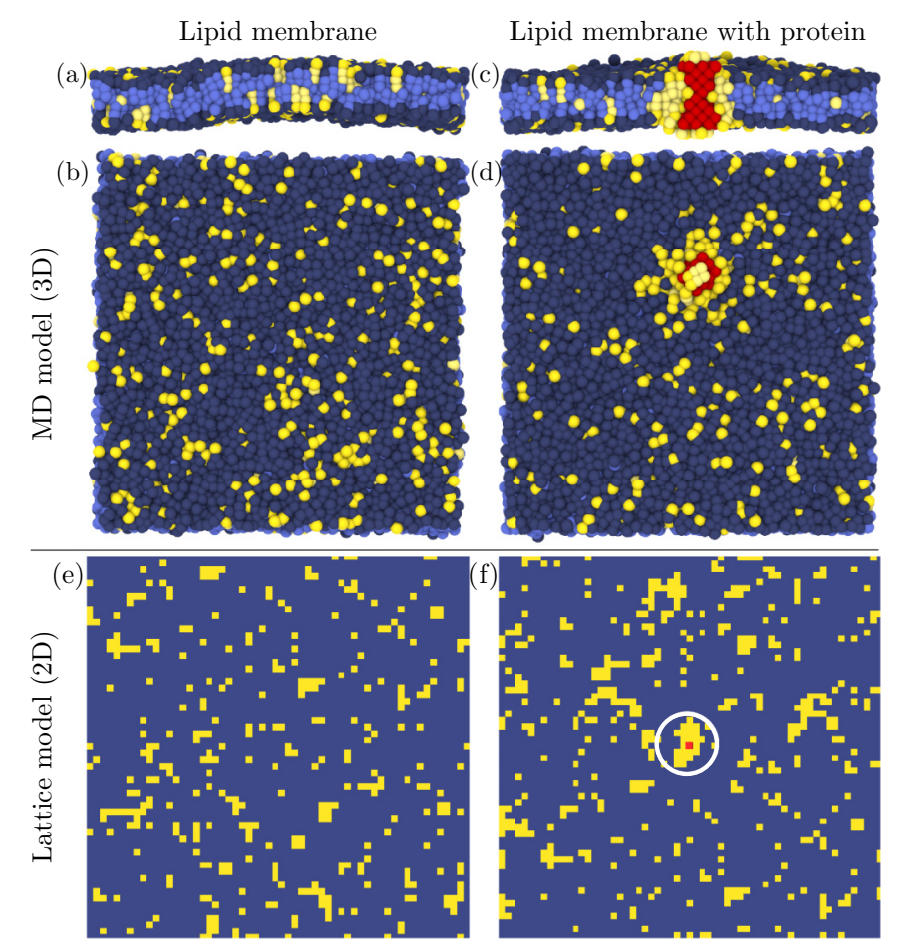

FIG. 2. Protein-driven formation of lipid raft domain in a binary mixture membrane in a mixed phase. (a) Lateral and (b) top view of the bilayer in a mixed phase simulated by coarse-grained MD model. Yellow beads represent raft lipids, blue beads represent non-raft lipids. Lipid heads are indicated with darker colors. (c) Side view of the protein (red) in MD simulations. (d) Top view of the lipid raft (cluster of yellow lipids) surrounding the protein (red) in MD simulations. (e) Top view of the lipid raft (cluster of yellow lipids) from the 2D Ising-like simulations, blue dots correspond to $\mathrm{N}$ lipids. (f) Top view of the lipid raft in 2D Ising-like simulations with the insertion of the protein (red dot) [66].

\section{B. Lattice model}

To mimic a two-dimensional cell membrane, we also use an Ising-based lattice model. The advantage of this approach, compared with the MD simulations studied in this paper, is the very low computational cost that enable us to explore a wide phase diagram on longer timescales. The membrane of the cell is represented by a two-dimensional square lattice of size $N \times N$ with spacing set to 1 . Simulations are performed on a lattice of size $N=128$. Periodic boundary conditions are applied in both dimensions. Each lattice site $c_{i}$ is occupied by either a lipid of type $\mathrm{R}$ or $\mathrm{N}$, in analogy with the spins in the Ising model that can have either state "up" or "down." Like in the Ising model, each lipid has pairwise interaction within a distance of one lattice spacing; therefore, it can only interact with the four-nearest-neighboring lipids (left, right, top, and bottom). The total energy of the system is represented by the Hamiltonian

$$
H\left(\left\{c_{i}\right\}_{i=1}^{N^{2}}\right)=\sum_{\langle i, j\rangle} E_{\mathrm{c}_{\mathrm{i}}, \mathrm{c}_{\mathrm{j}}},
$$

where the sum is over all nearest-neighbor pairs and $E_{\mathrm{c}_{\mathrm{i}}, \mathrm{c}_{\mathrm{j}}}$ is the energy of the interaction of the lipids on lattice sites $i$ and $j$ of type $c_{i}$ and $c_{j}$. Interaction between two lipids of the 
same type $(\mathrm{RR}$ or $\mathrm{NN})$ is attractive $\left(E_{\mathrm{RR}}=E_{\mathrm{NN}}<0\right)$, whereas the interaction among different type of lipids ( $\mathrm{RN}$ or NR) is repulsive $\left(E_{\mathrm{RN}}>0\right)$. The interaction energies are related by $E_{\mathrm{RR}}=E_{\mathrm{NN}}=-E_{\mathrm{RN}}$. The interaction strength is always given as the absolute value of the lipid-lipid interaction energy $\left|E_{\mathrm{RN}}\right|$ and in units of $\frac{1}{\beta}$ where $\beta=\left(k_{\mathrm{B}} T\right)^{-1}$.

We simulate the model by using Monte Carlo simulations with conserved dynamics $[67,68]$ so that each lipid can exchange its position with one of its four nearest neighbors. The exchange of two lipids from state $\left\{c_{i}\right\}$ to state $\left\{c_{i}^{\prime}\right\}$ follows the Glauber dynamics [69] and is executed with probability

$$
p(\Delta H)=\frac{1}{1+e^{\beta \Delta H}},
$$

where $\Delta H=H\left(\left\{c_{i}^{\prime}\right\}\right)-H\left(\left\{c_{i}\right\}\right)$ [69]. At equilibrium, the critical interaction strength for the Ising model is $\left|E_{\mathrm{RN}}\right|=$ $\frac{\ln (1+\sqrt{2})}{2} \approx 0.44$ [70]. Below the critical interaction strength, the lipids are in a mixed phase and above they phase separate into two domains. Here we focus on the mixed phase and therefore we limit the range of the interaction strength to

$$
\left|E_{\mathrm{RN}}\right| \leqslant 0.4 \text {. }
$$

A snapshot of the membrane in the mixed phase is reported in Fig. 2(e). In the following, a single Monte Carlo step corresponds to a sequence of $N^{2}$ updates of randomly selected nearest-neighbor pairs $[45,71,72]$.

To induce raft formation we add a protein in the membrane by replacing one lipid $\mathrm{R}$. The protein interacts with both $\mathrm{R}$ and $\mathrm{N}$ lipids, so we introduce the nearest-neighbor interaction energies $E_{\mathrm{RP}}$ and $E_{\mathrm{NP}}$. To induce a local aggregation of raft lipids, the protein is attractive to lipids of type $\mathrm{R}\left(E_{\mathrm{RP}}<0\right)$ and it is repulsive to lipids of type $\mathrm{N}\left(E_{\mathrm{NP}}>0\right)$. For simplicity, we chose the two interaction energies as $E_{\mathrm{RP}}=-E_{\mathrm{NP}}$ and impose

$$
\left|E_{\mathrm{RP}}\right| \geqslant\left|E_{\mathrm{RN}}\right|,
$$

which promotes clustering of $R$ lipids. The protein evolves according to the same dynamics of the lipids. A snapshot of the protein-driven raft formation in the lattice model is reported in Fig. 2(f): The protein, represented with a red square, is surrounded by a cluster of raft lipids (yellow) as highlighted by the white circle. The evolution dynamics of the protein driven lipid raft domain in the lattice model is shown in the Supplemental Movie S2 [66].

\section{RESULTS}

To clarify the effect of the presence of the protein on the raft formation, we investigate for both models the following quantities: (i) The size of aggregated raft lipid clusters around the protein, (ii) the dynamical behavior of the protein by MSD, and (iii) the real-space organization of the raft.

\section{A. Cluster size}

In the MD model, a cluster is defined as a set of connected raft lipids that fulfill the neighboring criterion, based on the mutual distance among all beads that form the lipids. In particular, two raft lipids belong to the same cluster if their distance is less than $1.2 \sigma$. After the addition of the transmembrane
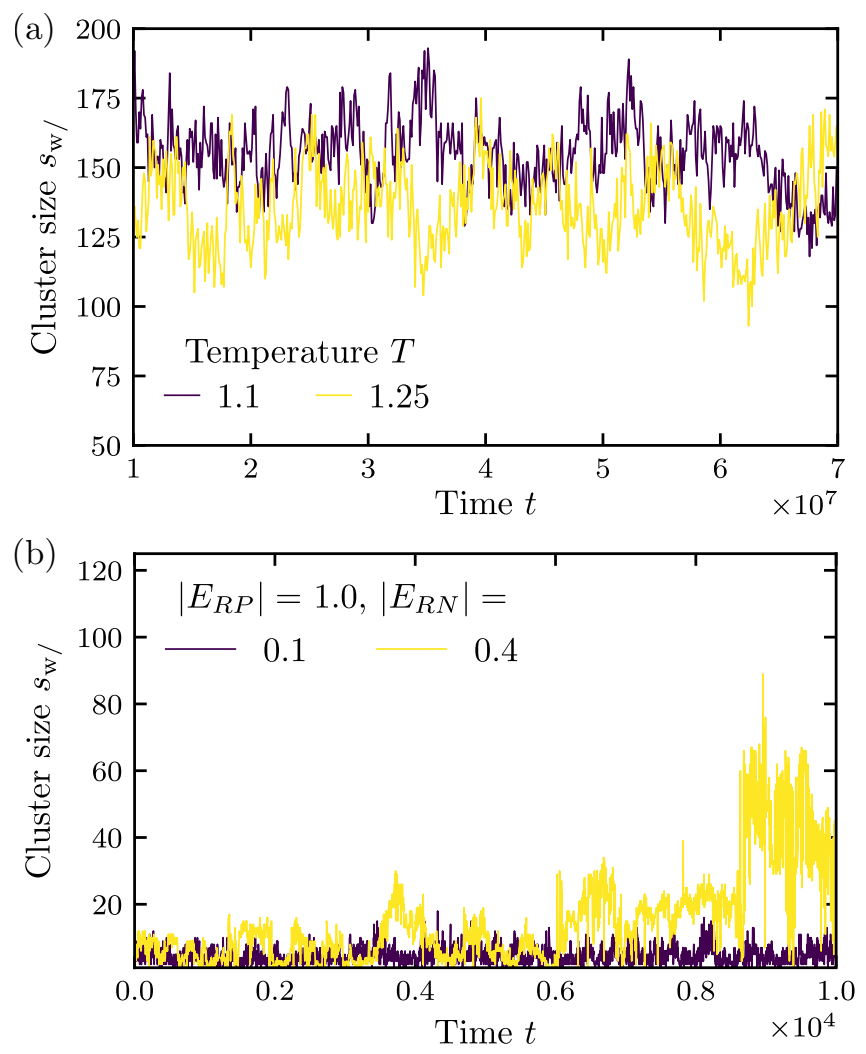

FIG. 3. Evolution of cluster size of lipid rafts with time. Both panels show the evolution of the cluster size of lipid rafts with("w/ protein") a protein versus time depending on the temperature $k_{\mathrm{B}} T / \epsilon$ and interaction strength $\left|E_{\mathrm{RN}}\right|$, respectively, for the (a) $\mathrm{MD}$ and (b) lattice model.

protein, raft lipids aggregate around it until the cluster size reaches a steady state.

Figure 3(a) shows the evolution of the cluster size with time as a function of two rescaled temperature $k_{\mathrm{B}} T / \epsilon$ for the MD model, while Fig. 3(b) shows the variation in time for different interaction strength $\left|E_{\mathrm{RN}}\right|$. The cluster size of the cluster containing the one obstacle $s_{w}$ / fluctuates strongly in time in the lattice model with respect to the MD simulations. The time needed for the cluster to grow in the lattice model gets longer with increasing $\left|E_{\mathrm{RP}}\right|$ and $\left|E_{\mathrm{RN}}\right|$. Specifically, we find that upon increasing $\left|E_{\mathrm{RN}}\right|$, the persistence time of the cluster grows.

Figure 4(a) shows the time-averaged cluster size as a function of the rescaled temperature $k_{\mathrm{B}} T / \epsilon$ : The cluster of lipids around the protein, indicated with the blue dot markers (labeled "w/ protein"), decreases with increasing temperatures. Raft lipids also clusterize without the assistance of the protein, but the cluster size is a factor of ten smaller, as indicated by the yellow squares (labeled as "without protein").

For the lattice model, two raft lipids are assigned to the same cluster if they are nearest neighbors on the lattice. Clusters are identified by using the Hoshen-Kopelmann algorithm for percolation [73]. In this model description, the size of clusters strongly fluctuates in time, and clusters are subjected to continuous formation and disruption, in analogy with experimental observation of fluctuating raft formation, as reported in recent experiments $[57,58]$. 

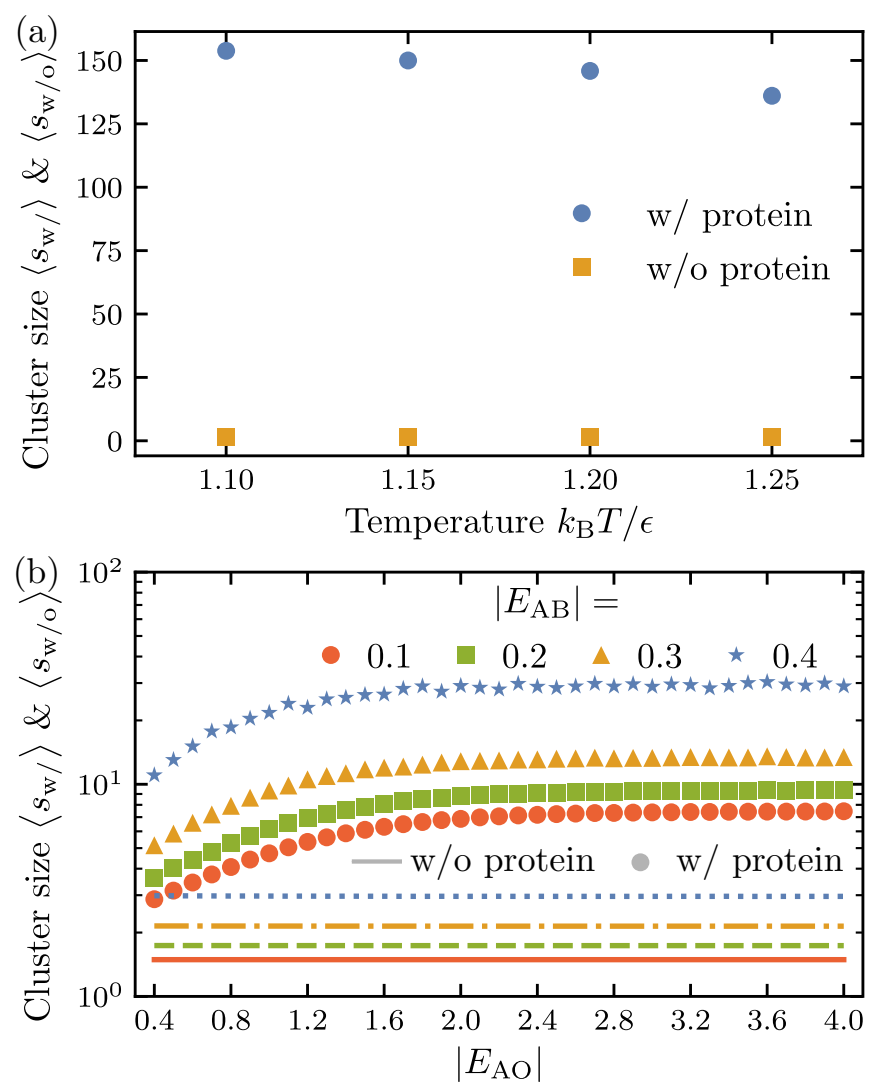

FIG. 4. Average cluster size of lipid rafts. Both panels show the average cluster size of lipid rafts with("w/ protein") and without("w/o protein") a protein depending on the temperature $k_{\mathrm{B}} T / \epsilon$ and interaction strength $\left|E_{\mathrm{RP}}\right|$, respectively, for the (a) MD and (b) lattice model.

In Fig. 4(b) after the addition of the protein, we observe that the clusters of lipids around the protein, indicated with blue markers ("w/ protein"), saturate with increasing interaction strength between the lipids and the protein $\left|E_{\mathrm{RP}}\right|$ : The size of clusters involving a protein increases up to maximum size at $\left|E_{\mathrm{RP}}\right| \approx 3$ and remains constant for stronger interaction strengths. With increasing interaction strengths between the lipids $\left|E_{\mathrm{RN}}\right|$, the average cluster size increases. For completeness, we also report the cluster size behavior of lipids without the presence of the protein, indicated with lines ("w/o protein").

Since the effective temperature $k_{\mathrm{B}} T / \epsilon$ scales as the inverse of the interaction strength $\left|E_{\mathrm{RN}}\right|$, the average cluster size decreases in both the MD model and the lattice model with increasing temperature, coherently confirming the trend on two different length scales and timescales.

\section{B. Diffusion}

The analysis of the mean squared displacement reveals information on the type of diffusion regimes and enable the determination of the diffusion constant. In this section we report the MSD of the protein to examine its mobility with the aggregated cluster. For both models, we focus on the protein movement within the two-dimensional surface of the lipid
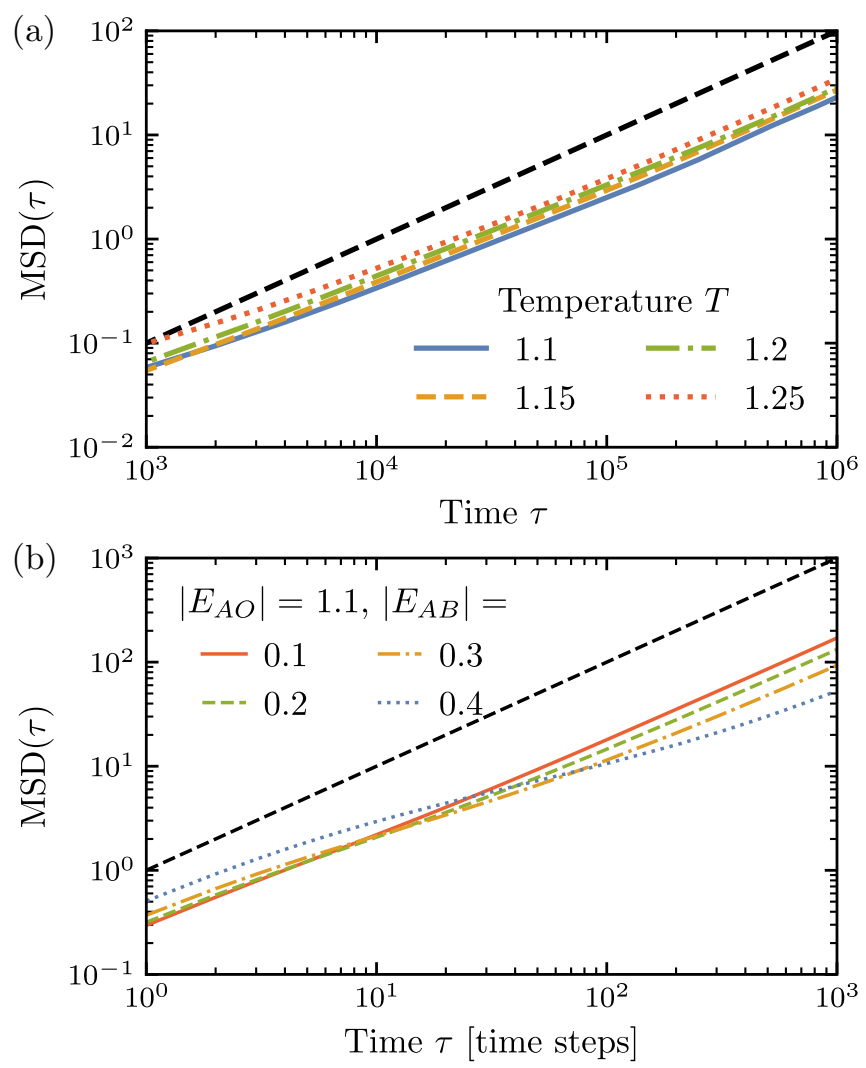

FIG. 5. Mean squared displacement of the protein. (a) In MD simulations, the protein diffuses in the liquid phase. (b) In the lattice model, at short timescales the protein diffuses in the cluster whereas at long timescales it diffuses with the whole cluster.

membrane. The MSD is defined as

$$
\operatorname{MSD}(\tau)=\left\langle|\vec{x}(t+\tau)-\vec{x}(t)|^{2}\right\rangle_{t}
$$

depending on the time increment $\tau$ where $\vec{x}(t)$ is the position in the membrane and $\langle\cdot\rangle_{t}$ is the average over time $t$. To calculate the MSD in Eq. (6), $t$ is chosen such that the membrane is at equilibrium, i.e., the average cluster size is stable. Figure 5 shows the MSD as a function of time for the MD model [Fig. 5(a)] and the lattice model [Fig. 5(b)].

In the MD simulations, the position of the protein is the center of mass in the $x y$ plane. Figure 5(a) shows that the behavior of MSD changes over time at various temperatures. Although the P mobility is suppressed with decreasing temperature, the overall motion is diffusive, confirming that the state point is in the liquid phase.

To determine the type of diffusion and the corresponding diffusion coefficient, we fit the data with the function

$$
\operatorname{MSD}(\tau)=4 D \tau^{2 H},
$$

where $H$ is the Hurst exponent, which characterizes the type of diffusion: $H=\frac{1}{2}$ is linear, $H<\frac{1}{2}$ is subdiffusive, and $H>\frac{1}{2}$ is superdiffusive behavior $[74,75]$. Notice that $D$ corresponds to the usual diffusion constant only for Brownian diffusion [76,77]. For the four investigated temperatures where the membrane is in the liquid phase, we find that a fit in the interval $\tau=10^{3}-10^{4}$ yields a subdiffusive regime because $\mathrm{H} \approx 0.4$. For $\tau=10^{5}-10^{6}, \mathrm{H} \approx 0.5$, pointing out 
that, in the long run, the diffusion is normal. As a matter of fact, the black dashed line is a reference line for the MSD curve expected for a linear diffusion process with diffusion constant $D=2.5 \times 10^{-5}$.

In the lattice model, after a transient regime the protein displays a diffusive behavior, in analogy of what has been observed in MD simulations. The crossover is ultimately ascribable to the different dynamical regimes followed by P: For short times the protein diffuses within the cluster, while on longer timescales the protein diffusion is that of the aggregated cluster. Attempting a linear fit of the asymptotic regime through the formula

$$
\operatorname{MSD}(\tau)=4 D \tau
$$

allows a systematic investigation of the $D$ dependence on the simulations parameters. Indeed, we report the MSD behavior with the interaction strength $\left|E_{\mathrm{RP}}\right|$ set to 1.1 and $\left|E_{\mathrm{RN}}\right|$ varied in Fig. 5(b). The black dashed line illustrates the MSD of a protein that does not interact with the lipids. Here the interaction strength among the lipids, $\left|E_{\mathrm{RN}}\right|$, is also set to zero. As the cluster size grows with increasing interaction strength $\left|E_{\mathrm{RN}}\right|$ [see Fig. 4(b)], the diffusion constant decreases.

\section{Components of the diffusion constant}

In the conventional description of Brownian motion, the diffusion constant is proportional to the inverse mass which here is quantified by the cluster size. We therefore assume $D \sim\left\langle s_{\mathrm{w}}\right\rangle^{-1}$ and introduce the following fitting function:

$$
D\left(\left\langle s_{\mathrm{w} /}\right\rangle\right)=\frac{1}{a\left\langle s_{\mathrm{w} /}\right\rangle+b},
$$

where $a$ is the angular coefficient and $b$ is the offset. For the sake of clarity, Fig. 6(a) shows the inverse diffusion constant as a function of the average cluster size. For different values of the interaction strength $\left|E_{\mathrm{RP}}\right| \in\{0.6,0.8,1.0,1.2,1.4\}$, indicated by different markers that indicate the simulation data and the solid line is the fit. The fit works quite well for all interaction strengths $\left|E_{\mathrm{RP}}\right|<1$.5. Figure 6(b) shows the offset $b$ as function of the interaction strength $\left|E_{\mathrm{RP}}\right|$. For $\left|E_{\mathrm{RP}}\right|<1.4$, the offset is quite constant around four.

The offset means that the diffusion also has a constant part that is not influenced by the cluster size of the cluster with the protein. For weaker interaction strengths $\left|E_{\mathrm{RP}}\right|$, the offset becomes important since the cluster size decreases, as well as the slope $a$. So if the interaction strength goes to $\left|E_{\mathrm{RP}}\right| \rightarrow 0$ and $s \rightarrow 0$, only the constant is left and the inverse diffusion constant become $\frac{1}{D}=4$. By inverting this, we get that the diffusion constant is $D=\frac{1}{4}$, which is the diffusion of a nonfunctional protein.

Hence, we conclude that the diffusion process has two contributions: One that is constant and is related to the free diffusion of the protein in the cluster, while the second depends on the cluster size and is due to the cluster diffusing in the system.

\section{Real-space distribution}

Real-space distribution functions give us information about the variation in density relative to a reference point which

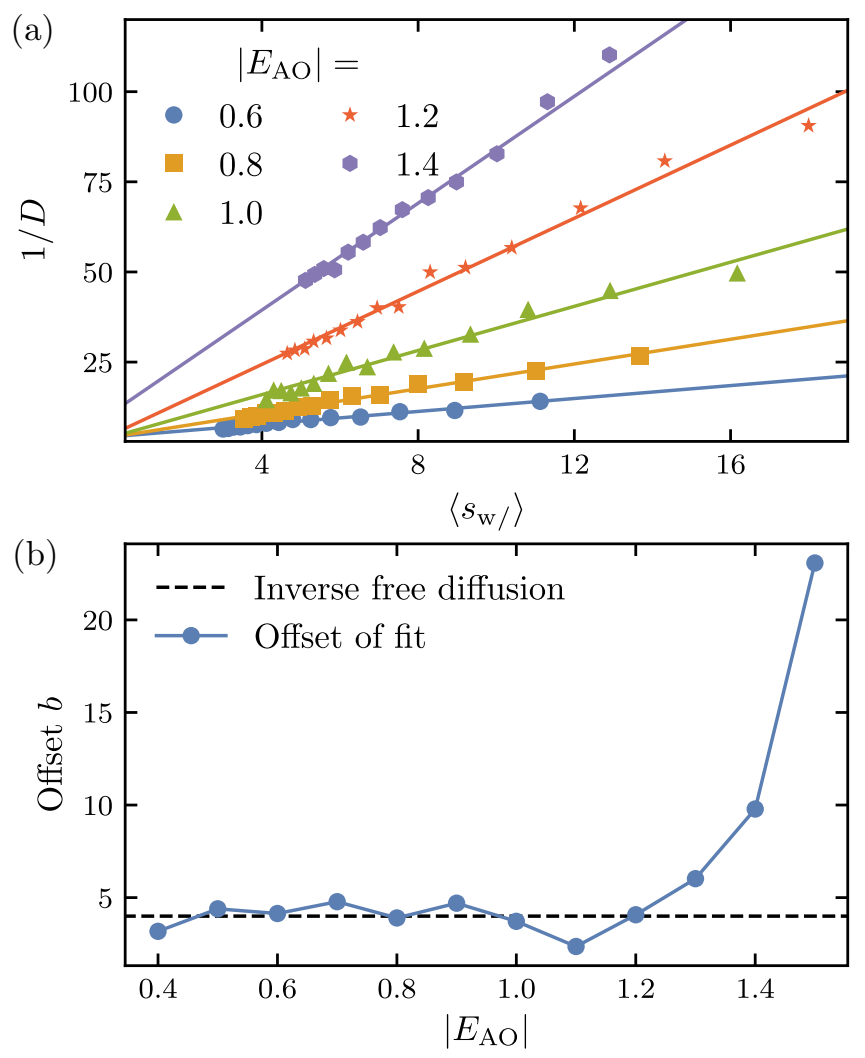

FIG. 6. Diffusion constant analysis for the lattice model. (a) Inverse diffusion constant as a function of cluster size for fixed interaction strengths $\left|E_{\mathrm{RP}}\right|$ showing a linear behavior (lines represent linear fits). The fitting function is $1 / D_{\text {fit }}\left(\left\langle s_{\mathrm{w} /}\right\rangle\right)=a\left\langle s_{\mathrm{w} /}\right\rangle+b$. (b) Offset $b$ of the fitted curves presented in panel (a) as a function of $\left|E_{\mathrm{RP}}\right|$. For $\left|E_{\mathrm{RP}}\right|<1.5$, the offset is quite constant around four, which corresponds to the inverse of the diffusion constant of a noninteracting protein $\left(\left|E_{\mathrm{RN}}\right|=0\right.$ and $\left.\left|E_{\mathrm{RP}}\right|=0\right)$.

is the protein in our models. Here, we focus on the radial distribution function (RDF), which indicates the density of raft lipids depending on the distance to the protein. In general, we normalize the RDF by a homogeneous distribution of raft lipids according to the respective lipid ratios, so that values greater (smaller) than 1 indicate higher (lower) density than an equilibrated system. Figure 7(a) shows the RDF of raft lipids relative to the center of mass of the protein in the $x y$ plane for the MD model. Considering the membrane to be equilibrated with a stable cluster, we report the time-average RDF. We note that, when decreasing the temperature, the tail of the RDF increases, which is consistent with the increase of the average cluster size observed in Fig. 4(a).

Figure 7(b) shows the RDF of raft lipids around the protein for a fixed interaction strength $\left|E_{\mathrm{RP}}\right|=1.1$. The distribution function shows a high density close to the protein which decays toward the value 1 . With increasing interaction strength $\left|E_{\mathrm{RN}}\right|$, the maximum density close to the protein increases, in agreement with the results shown in Fig. 4(b).

\section{CONCLUSIONS}

Lipid rafts are microdomains on the plasma membrane enriched in cholesterol, sphingolipids, and GPI-anchored pro- 

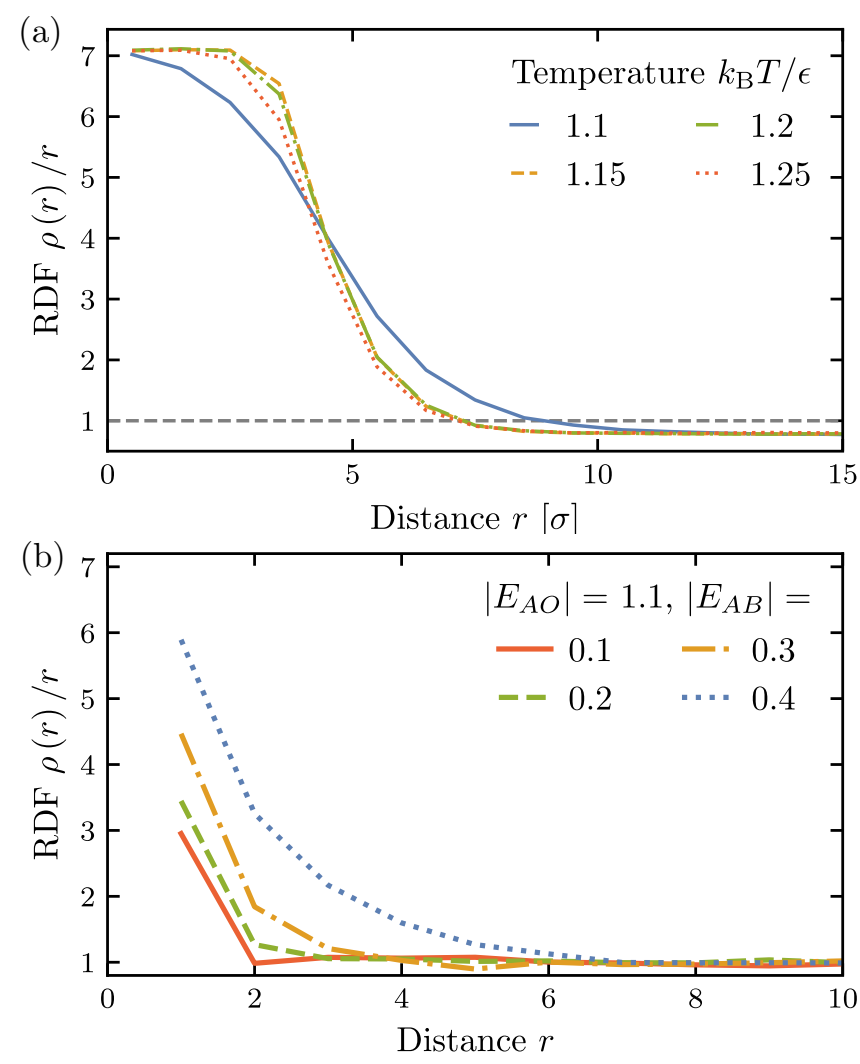

FIG. 7. Time-averaged RDF of lipids around the protein. (a) MD simulations and (b) lattice model. With (a) decreasing temperature or (b) increasing attraction $\left|E_{\mathrm{RN}}\right|$, the tail of RDF grows, reflecting that the size of the raft is increasing.

teins that play a crucial role in many cellular processes, such as signal transduction, membrane trafficking, and pathogen entry. Moreover, the aggregation of these microdomains allows us to display a cellular response in a very short time. The mechanisms underlying rapid raft formation of have been long debated [3].

In this paper, we study the role of a transmembrane protein on lipid raft formation by means of coarse-grained moleculardynamics simulations and an Ising-model-based approach. Our results show that the protein triggers the lipid aggregation in the form of a raft, in agreement with experiments [57,58]. Raft formation in our model occurs in the disordered phase and does not reflect stable phase separation between raft lipids. From the point of view of statistical mechanics of phase transitions, lipid rafts could be seen as droplets of the raft lipids in the mixed phase. In the Ising model, those droplets would be the size of the correlation length $\xi$ which becomes large close to the critical point, but would be very short lived. The action of the transmembrane protein is analogous to a localized magnetic field in the Ising model, which help nucleate and stabilize the droplet.

Our model is thus in agreement with experimental observations in living cells suggesting that lipid raft are dynamic structures rather than stable domains as observed in synthetic lipid membranes [3]. Our results also show that the size of the cluster of raft lipids surrounding the protein reaches a steady state and decreases: (i) With increasing the temperature in the MD model (ii) with the inverse of the interaction strength (which we can consider as a proxy for temperature) in the lattice simulations. Note that our results do not strictly depend on the concentration of the phases as long as the parameters of the system place it at the boundary of the mixed phase. The cluster sizes will only be rescaled by a concentrationdependent factor, but the general phenomenology we describe should remain unchanged.

Furthermore, from MSD analysis we confirm that the overall motion of the protein is diffusive in both models. The diffusion constant depends on the size of the raft domain, indicating that the raft and and the protein move coherently as a single object. The results obtained combining two complementing simulation methods tackling different length scales and timescales coherently support a protein-driven lipid-raftformation model.

Our model treats interactions between lipids in a generic way and could thus be applied to lipid rafts but also to other cases. For instance, evidence of aggregation of lipids around a protein forming a ring or annular shell has been reported by electron-spin resonance (ESR) studies since the late seventies [78-82] and might also be described by our model.

\section{ACKNOWLEDGMENTS}

We thank F. Brauns, C.-F. Chou, E. Frey, R. Guerra, C. Negri, and A. L. Sellerio for useful discussions. M.H. was supported by the European Commission under ERASMUS+ program thanks to a joint agreement between the University of Milan and Ludwig Maximilian University Munich.

M.H. and S.B. performed numerical simulations and wrote the paper. M.H., S.B., and A.T. analyzed the data. C.A.M.L.P. and S.Z. designed and coordinated the project.
[1] O. S. Andersen and R. E. Koeppe, Annu. Rev. Biophys. Biomol. Struct. 36, 107 (2007).

[2] N. Ramakrishnan, P. B. Sunil Kumar, and R. Radhakrishnan, Phys. Rep. 543, 1 (2014).

[3] E. Sezgin, I. Levental, S. Mayor, and C. Eggeling, Nat. Rev. Mol. Cell Biol. 18, 361 (2017).

[4] D. Brown and E. London, Annu. Rev. Cell Dev. Biol. 14, 111 (1998).

[5] R. G. Anderson and K. Jacobson, Science 296, 1821 (2002).

[6] K. Simons and E. Ikonen, Nature (London) 387, 569 (1997).
[7] D. Lingwood and K. Simons, Science 327, 46 (2010).

[8] T. Harder and K. Simons, Curr. Opin. Cell Biol. 9, 534 (1997).

[9] R. Varma and S. Mayor, Nature (London) 394, 798 (1998).

[10] R. E. Brown, J. Cell Sci. 111, 1 (1998).

[11] D. A. Brown and E. London, J. Biol. Chem. 275, 17221 (2000).

[12] A. D. Douglass and R. D. Vale, Cell (Cambridge, MA, U. S.) 121, 937 (2005).

[13] L. J. Pike, J. Lipid Res. 47, 1597 (2006). 
[14] D. M. Owen, D. J. Williamson, A. Magenau, and K. Gaus, Nat. Commun. 3, 1256 (2012).

[15] M. Schulz, A. Olubummo, and W. H. Binder, Soft Matter 8, 4849 (2012).

[16] A. Honigmann, V. Mueller, H. Ta, A. Schoenle, E. Sezgin, S. W. Hell, and C. Eggeling, Nat. Commun. 5, 5412 (2014).

[17] J. H. Lorent, B. Diaz-Rohrer, X. Lin, K. Spring, A. A. Gorfe, K. R. Levental, and I. Levental, Nat. Commun. 8, 1219 (2017).

[18] E. Sezgin and P. Schwille, Cold Spring Harbor Perspect. Biol. 3, a009803 (2011).

[19] A. S. Klymchenko and R. Kreder, Chem. Biol. 21, 97 (2014).

[20] C. Eggeling, Essays Biochem. 57, 69 (2015).

[21] J. Ortega-Arroyo and P. Kukura, Phys. Chem. Chem. Phys. 14, 15625 (2012).

[22] A. Kusumi, C. Nakada, K. Ritchie, K. Murase, K. Suzuki, H. Murakoshi, R. S. Kasai, J. Kondo, and T. Fujiwara, Annu. Rev. Biophys. Biomol. Struct. 34, 351 (2005).

[23] J. C. Chang and S. J. Rosenthal, ACS Chem. Neurosci. 3, 737 (2012).

[24] K. G. Suzuki, in Lipid Signaling Protocols (Springer, New York, 2016), pp. 229-238.

[25] R. F. de Almeida, L. M. Loura, A. Fedorov, and M. Prieto, J. Mol. Biol. 346, 1109 (2005).

[26] A. J. Fijałkowski, Wikimedia Commons (2006), https:// en.wikipedia.org/wiki/Lipid_raft?\#/media/File:Lipid_raft_ organisation_scheme.svg.

[27] K. Simons and W. L. Vaz, Annu. Rev. Biophys. Biomol. Struct. 33, 269 (2004).

[28] K. Simons and M. J. Gerl, Nat. Rev. Mol. Cell Biol. 11, 688 (2010).

[29] L. K. Tamm and H. M. McConnell, Biophys. J. 47, 105 (1985).

[30] S. L. Veatch and S. L. Keller, Biophys. J. 85, 3074 (2003).

[31] N. Kahya, D. A. Brown, and P. Schwille, Biochemistry 44, 7479 (2005).

[32] I. Levental, F. J. Byfield, P. Chowdhury, F. Gai, T. Baumgart, and P. A. Janmey, Biochem. J. 424, 163 (2009).

[33] J. F. Hancock, Nat. Rev. Mol. Cell Biol. 7, 456 (2006).

[34] I. Levental, M. Grzybek, and K. Simons, Proc. Natl. Acad. Sci. U. S. A. 108, 11411 (2011).

[35] A. Tian and T. Baumgart, Biophys. J. 96, 2676 (2009).

[36] M. Heinrich, A. Tian, C. Esposito, and T. Baumgart, Proc. Natl. Acad. Sci. U. S. A. 107, 7208 (2010).

[37] R. Parthasarathy, C.-H. Yu, and J. T. Groves, Langmuir 22, 5095 (2006).

[38] I. R. Cooke and M. Deserno, Biophys. J. 91, 487 (2006).

[39] H. J. Risselada and S. J. Marrink, Phys. Chem. Chem. Phys. 11, 2056 (2009).

[40] B. Różycki, T. R. Weikl, and R. Lipowsky, Phys. Rev. Lett. 100, 098103 (2008).

[41] A. R. Honerkamp-Smith, P. Cicuta, M. D. Collins, S. L. Veatch, M. den Nijs, M. Schick, and S. L. Keller, Biophys. J. 95, 236 (2008).

[42] S. L. Veatch, P. Cicuta, P. Sengupta, A. Honerkamp-Smith, D. Holowka, and B. Baird, ACS Chem. Biol. 3, 287 (2008).

[43] A. R. Honerkamp-Smith, S. L. Veatch, and S. L. Keller, Biochim. Biophys. Acta, Biomembr. 1788, 53 (2009).

[44] B. B. Machta, S. L. Veatch, and J. P. Sethna, Phys. Rev. Lett. 109, 138101 (2012).
[45] A. R. Honerkamp-Smith, B. B. Machta, and S. L. Keller, Phys. Rev. Lett. 108, 265702 (2012).

[46] Y.-J. Chen, N. M. Paquette, B. B. Machta, and J. P. Sethna, arXiv:1307.6899.

[47] E. D. Mitra, S. C. Whitehead, D. Holowka, B. Baird, and J. P. Sethna, J. Phys. Chem. B 122, 3500 (2018).

[48] G. Gompper and M. Schick, Phys. Rev. B 41, 9148 (1990).

[49] G. W. Feigenson and J. T. Buboltz, Biophys. J. 80, 2775 (2001).

[50] J. R. Silvius, Biophys. J. 85, 1034 (2003).

[51] A. Bunge, P. Müller, M. Stöckl, A. Herrmann, and D. Huster, Biophys. J. 94, 2680 (2008).

[52] G. R. Chichili and W. Rodgers, Cell. Mol. Life Sci. 66, 2319 (2009).

[53] B. B. Machta, S. Papanikolaou, J. P. Sethna, and S. L. Veatch, Biophys. J. 100, 1668 (2011).

[54] B. P. Head, H. H. Patel, and P. A. Insel, Biochim. Biophys. Acta, Biomembr. 1838, 532 (2014).

[55] F. Schneider, D. Waithe, M. P. Clausen, S. Galiani, T. Koller, G. Ozhan, C. Eggeling, and E. Sezgin, Mol. Biol. Cell 28, 1507 (2017).

[56] I. R. Cooke, K. Kremer, and M. Deserno, Phys. Rev. E 72, 011506 (2005).

[57] N. Komura, K. G. N. Suzuki, H. Ando, M. Konishi, M. Koikeda, A. Imamura, R. Chadda, T. K. Fujiwara, H. Tsuboi, R. Sheng, W. Cho, K. Furukawa, K. Furukawa, Y. Yamauchi, H. Ishida, A. Kusumi, and M. Kiso, Nat. Chem. Biol. 12, 402 (2016).

[58] K. G. Suzuki, H. Ando, N. Komura, M. Konishi, A. Imamura, H. Ishida, M. Kiso, T. K. Fujiwara, and A. Kusumi, in Methods in Enzymology, Chemical Glycobiology Part B. Monitoring Glycans and their Interactions, edited by B. Imperiali (Academic Press, 2018), Vol. 598, pp. 267-282.

[59] F. W. Starr, B. Hartmann, and J. F. Douglas, Soft Matter 10, 3036 (2014).

[60] H. C. Andersen, J. D. Weeks, and D. Chandler, Phys. Rev. A 4, 1597 (1971).

[61] S. Plimpton, J. Comp. Phys. 117, 1 (1995).

[62] https://github.com/ComplexityBiosystems/Flat_Membrane_ with_Protein_MD_Simulations, accessed on September 9, 2019.

[63] I. R. Cooke and M. Deserno, J. Chem. Phys. 123, 224710 (2005).

[64] S. Tristram-Nagle and J. F. Nagle, Chem. Phys. Lipids 127, 3 (2004).

[65] J. W. Klingelhoefer, T. Carpenter, and M. S. Sansom, Biophys. J. 96, 3519 (2009).

[66] See Supplemental Material at http://link.aps.org/supplemental/ 10.1103/PhysRevE.100.042410 for videos from lattice model and MD simulations showing a flat membrane in the mixed phase where a protein induces the formation of clusters.

[67] P. Krapivsky, S. Redner, and E. Ben-Naim, A Kinetic View of Statistical Physics (Cambridge University Press, Cambridge, 2010).

[68] K. Kawasaki, Phys. Rev. 145, 224 (1966).

[69] R. J. Glauber, J. Math. Phys. 4, 294 (1963).

[70] R. J. Baxter, Exactly Solved Models in Statistical Mechanics (Academic Press, London, 1982).

[71] M. Plischke and B. Bergersen, Equilibrium Statistical Physics (World Scientific, Singapore, 2006).

[72] N. Metropolis, A. W. Rosenbluth, M. N. Rosenbluth, A. H. Teller, and E. Teller, J. Chem. Phys. 21, 1087 (1953). 
[73] J. Hoshen and R. Kopelman, Phys. Rev. B 14, 3438 (1976).

[74] H. E. Hurst, Trans. Amer. Soc. Civil Eng. 116, 770 (1951).

[75] B. B. Mandelbrot and J. W. Van Ness, SIAM Rev. 10, 422 (1968).

[76] R. Metzler and J. Klafter, Phys. Rep. 339, 1 (2000).

[77] A. Taloni, A. Chechkin, and J. Klafter, Phys. Rev. Lett. 104, 160602 (2010).

[78] A. Lee, Trends Biochem. Sci. 2, 231 (1977).
[79] A. Watts, I. D. Volotovski, and D. Marsh, Biochemistry 18, 5006 (1979).

[80] A. Watts, J. Davoust, D. Marsh, and P. Devaux, Biochim. Biophys. Acta, Biomembr. 643, 673 (1981).

[81] J. Davoust and P. F. Devaux, J. Magn. Reson. (1969-1992) 48, 475 (1982).

[82] B. Sternberg, C. L'Hostis, C. A. Whiteway, and A. Watts, Biochim. Biophys. Acta, Biomembr. 1108, 21 (1992). 